\title{
HANKEL OPERATORS ON THE BERGMAN SPACE OF BOUNDED SYMMETRIC DOMAINS
}

\author{
KEHE ZHU
}

\begin{abstract}
Let $\Omega$ be a bounded symmetric domain in $\mathbb{C}^{n}$ with normalized volume measure $d V$. Let $P$ be the orthogonal projection from $L^{2}(\Omega, d V)$ onto the Bergman space $L_{a}^{2}(\Omega)$ of holomorphic functions in $L^{2}(\Omega, d V)$. Let $\bar{P}$ be the orthogonal projection from $L^{2}(\Omega, d V)$ onto the closed subspace of antiholomorphic functions in $L^{2}(\Omega, d V)$. The "little" Hankel operator $h_{f}$ with symbol $f$ is the operator from $L_{a}^{2}(\Omega)$ into $L^{2}(\Omega, d V)$ defined by $h_{f} g=$ $\bar{P}(f g)$. We characterize the boundedness, compactness, and membership in the Schatten classes of the Hankel operators $h_{f}$ in terms of a certain integral transform of the symbol $f$. These characterizations are further studied in the special cases of the open unit ball and the poly-disc in $\mathbb{C}^{n}$.
\end{abstract}

\section{INTRODUCTION}

Let $\Omega$ be a bounded symmetric domain in $\mathbb{C}^{n}$ with (normalized) volume measure $d V$. For any $1 \leq p<+\infty$, the Bergman space $L_{a}^{p}(\Omega)$ consists of holomorphic functions in $L^{p}(\Omega, d V)$. It is easy to see that $L_{a}^{p}(\Omega)$ is a closed subspace of $L^{p}(\Omega, d V)$. In particular, there exists an orthogonal projection

$$
P: L^{2}(\Omega, d V) \rightarrow L_{a}^{2}(\Omega) .
$$

Let $K(z, w)$ be the Bergman kernel of $\Omega$, then

$$
P f(z)=\int_{\Omega} K(z, w) f(w) d V(w) .
$$

The above integral formula extends $P$ to $L^{1}(\Omega, d V)$. Let $\bar{P}$ be the orthogonal projection from $L^{2}(\Omega, d V)$ onto $\overline{L_{a}^{2}(\Omega)}=\left\{\bar{f}: f \in L_{a}^{2}(\Omega)\right\}$, then

$$
\begin{aligned}
\bar{P} f(z) & =\int_{\Omega} \overline{K(z, w)} f(w) d V(w) \\
& =\int_{\Omega} K(w, z) f(w) d V(w) .
\end{aligned}
$$

This formula also extends $\bar{P}$ to $L^{1}(\Omega, d V)$.

Received by the editors March 29, 1989.

1980 Mathematics Subject Classification (1985 Revision). Primary 47B35, 47B10; Secondary 47B38, 47B05.

Research supported by the National Science Foundation. 
Given $\phi \in L^{2}(\Omega, d V)$, define two operators $H_{\phi}$ and $h_{\phi}$ with domain $L_{a}^{2}(\Omega)$ as follows:

$$
H_{\phi} f=(I-P)(\phi f), \quad h_{\phi} f=\bar{P}(\phi f),
$$

where $I$ is the identity operator. $H_{\phi}$ is called the Hankel operator with symbol $\phi$ and $h_{\phi}$ is called the reduced (or little) Hankel operator with symbol $\phi$. The word "reduced" (or little) is justified by the inequality

$$
\bar{P}-P_{0} \leq I-P,
$$

where $P_{0}$ is the orthogonal projection (rank 1) from $L^{2}(\Omega, d V)$ onto the constants, that is

$$
P_{0} f(z)=\int_{\Omega} f(z) d V(z) .
$$

The main problems that we are interested in here are the following:

Problem 1. When are $h_{\phi}$ and $H_{\phi}$ bounded operators from $L_{a}^{2}(\Omega)$ into $L^{2}(\Omega, d V)$ ?

Problem 2. When are $h_{\phi}$ and $H_{\phi}$ compact?

Problem 3. When are $h_{\phi}$ and $H_{\phi}$ in the Schatten-Von Neumann ideals $S_{p}$ ?

An immediate consequence of the inequality $\bar{P}-P_{0} \leq I-P$ is that $H_{\phi}$ is bounded implies $h_{\phi}$ is bounded; $H_{\phi}$ is compact implies $h_{\phi}$ is compact; and $H_{\phi}$ is in $S_{p}$ implies $h_{\phi}$ is in $S_{p}$.

By now it is fairly well-known $[2,3]$ that the properties of $H_{\phi}$ and $H_{\bar{\phi}}$ are governed by the Berezin transform. More specifically, for any $f$ in $L^{1}(\Omega, d V)$, the Berezin transform of $f$ is the function $B f$ defined on $\Omega$ by

$$
B f(z)=\int_{\Omega} f(w)\left|k_{z}(w)\right|^{2} d V(w),
$$

where

$$
k_{z}(w)=\frac{K(w, z)}{\sqrt{K(z, z)}}
$$

is a unit vector in $L_{a}^{2}(\Omega)$ for each $z \in \Omega$. For any $\phi \in L^{2}(\Omega, d V)$, let

$$
M O(\phi)(z)=\left[B\left(|\phi|^{2}\right)(z)-|B \phi(z)|^{2}\right]^{1 / 2}, \quad z \in \Omega .
$$

$M O(\phi)$ describes a type of mean oscillation of $\phi$ in $\Omega[2,3]$. It was shown in [3] that

(1) $H_{\phi}, H_{\bar{\phi}}$ are bounded $\Leftrightarrow M O(\phi) \in B \mathbb{C}$;

(2) $H_{\phi}, H_{\bar{\phi}}$ are compact $\Leftrightarrow M O(\phi) \in \mathbb{C}_{0}$.

Here $B \mathbb{C}$ is the space of bounded continuous functions on $\Omega$ and $\mathbb{C}_{0}$ is the space of continuous functions on $\Omega$ vanishing on the boundary. 
In view of the above results, we see that the Berezin transform carries a lot of information on the Hankel operators $H_{\phi}$. The main purpose of this article is to demonstrate that there exists a similar integral transform on $\Omega$ which carries a lot of information on the reduced Hankel operators. We give a unified treatment on the size estimates of $h_{\phi}$ using the integral transform $V$ defined below. It should be pointed out that [6] gives a fairly complete theory on reduced Hankel operators. However, the theory in [6] does not cover our results here because Hankel operators (forms) in [6] are defined on a different space (or at least for the main results there), namely, the space of square integrable holomorphic functions with respect to the measure $K(z, z)^{-1} d V(z)$.

The integral transform $V$ is defined as follows. Given $f$ in $L^{1}(\Omega, d V)$, $V f$ is the function on $\Omega$ defined by

$$
V f(z)=\lambda_{\Omega} \int_{\Omega} f(w) \overline{k_{z}^{2}(w)} d V(w), \quad z \in \Omega,
$$

where

$$
\lambda_{\Omega}^{-1}=\int_{\Omega} K(z, z)^{-1} d V(z)
$$

Note that in the definition of the Berezin transform we used $\left|k_{z}(w)\right|^{2}$, while in the definition of $V$ we used $\overline{k_{z}^{2}(w)}$.

Theorem. Let $\Omega$ be any bounded symmetric domain in $\mathbb{C}^{n}$. If $\phi \in L^{2}(\Omega, d V)$ and $p \geq 1$, then

(1) $h_{\bar{\phi}}$ is bounded $\Leftrightarrow V \phi \in B \mathbb{C}$;

(2) $h_{\bar{\phi}}$ is compact $\Leftrightarrow V \phi \in \mathbb{C}_{0}$;

(3) $h_{\bar{\phi}}$ is in $S_{p} \Leftrightarrow V \phi \in L^{p}(d \lambda)$, where $d \lambda(z)=K(z, z) d V(z)$.

Note that for $\phi \in L^{2}(\Omega, d V)$, we always have $h_{\bar{\phi}}=h_{\overline{P \phi}}$, where $P$ is the Bergman projection. Thus in considering reduced Hankel operators, we can content ourselves with antiholomorphic symbols.

When $\phi$ is holomorphic in $\Omega, V \phi$ is "computable" in the most interesting cases. If $\Omega=B_{n}$ and $\phi$ is holomorphic in $B_{n}$, then we will show that $V \phi$ behaves like the functions

$$
\left(1-|z|^{2}\right)^{n+1} \frac{\partial^{n+1}}{\partial z^{\alpha}}(z) \quad(|\alpha|=n+1),
$$

where $\alpha=\left(\alpha_{1}, \ldots, \alpha_{n}\right)$ is an ordered $n$-tuple of nonnegative integers, $|\alpha|=$ $\alpha_{1}+\cdots+\alpha_{n}$, and

$$
\frac{\partial^{n+1} \phi}{\partial z^{\alpha}}(z)=\frac{\partial^{n+1} \phi}{\partial z_{1}^{\alpha_{1}} \cdots \partial z_{n}^{\alpha_{n}}}(z)
$$

for $|\alpha|=n+1$. Therefore, we obtain

Corollary 1 [6]. If $\phi$ is holomorphic in $B_{n}$ and $p \geq 1$, then

(1) $h_{\bar{\phi}}$ is bounded $\Leftrightarrow\left(1-|z|^{2}\right)^{n+1} \frac{\partial^{n+1} \phi}{\partial z^{\alpha}}(z) \in B \mathbb{C}$ for all $|\alpha|=n+1$; 
(2) $h_{\bar{\phi}}$ is compact $\Leftrightarrow\left(1-|z|^{2}\right)^{n+1} \frac{\partial^{n+1} \phi}{\partial z^{\alpha}}(z) \in \mathbb{C}_{0}$ for all $|\alpha|=n+1$;

(3) $h_{\bar{\phi}}$ is in $S_{p} \Leftrightarrow\left(1-|z|^{2}\right)^{n+1} \frac{\partial^{n+1} \phi}{\partial z^{\alpha}}(z) \in L^{p}(d \lambda)$ for all $|\alpha|=n+1$.

There is another case when we can "compute" $V \phi$ for $\phi$ holomorphic, namely, when $\Omega=\mathbb{D}^{n}$, the poly-disc in $\mathbb{C}^{n}$. For simplicity of notation, we will confine ourselves to the bidisc $\mathbb{D}^{2}$. In this case, we show that $V \phi$ is determined by the following five functions:

$$
\begin{aligned}
& T_{1} \phi\left(z_{1}\right)=\left(1-\left|z_{1}\right|^{2}\right)^{2} \frac{\partial^{2} \phi}{\partial z_{1}^{2}}\left(z_{1}, 0\right), \quad T_{2} \phi\left(z_{2}\right)=\left(1-\left|z_{2}\right|^{2}\right)^{2} \frac{\partial^{2} \phi}{\partial z_{2}^{2}}\left(0, z_{2}\right), \\
& T_{3} \phi\left(z_{1}\right)=\left(1-\left|z_{1}\right|^{2}\right)^{2} \frac{\partial^{3} \phi}{\partial z_{1}^{2} \partial z_{2}}\left(z_{1}, 0\right), \\
& T_{4} \phi\left(z_{2}\right)=\left(1-\left|z_{2}\right|^{2}\right) \frac{\partial^{3} \phi}{\partial z_{1} \partial z_{2}^{2}}\left(0, z_{2}\right), \\
& T \phi\left(z_{1}, z_{2}\right)=\left(1-\left|z_{1}\right|^{2}\right)^{2}\left(1-\left|z_{2}\right|^{2}\right)^{2} \frac{\partial^{4} \phi}{\partial z_{1}^{2} \partial z_{2}^{2}}\left(z_{1}, z_{2}\right) .
\end{aligned}
$$

It is possible to use lower order derivatives here (as well as in the case of $B_{n}$ ) if we only consider $S_{p}$ for $p>1[10,12]$.

Corollary 2. If $p \geq 1$ and $\phi$ is holomorphic in $\mathbb{D}^{2}$, then we have

(1) $h_{\bar{\phi}}$ is bounded $\Leftrightarrow T_{i} \phi \in B \mathbb{C}(\mathbb{D}) \quad(1 \leq i \leq 4), \quad T \phi \in B \mathbb{C}\left(\mathbb{D}^{2}\right)$;

(2) $h_{\bar{\phi}}$ is compact $\Leftrightarrow T_{i} \phi \in \mathbb{C}_{0}(\mathbb{D}) \quad(1 \leq i \leq 4), \quad T \phi \in \mathbb{C}_{0}\left(\mathbb{D}^{2}\right)$;

(3) $h_{\bar{\phi}}$ is in $S_{p} \Leftrightarrow T_{i} \phi \in L^{p}(\mathbb{D}, d \lambda) \quad(1 \leq i \leq 4), \quad T \phi \in L^{p}\left(\mathbb{D}^{2}, d \lambda\right)$.

\section{BASIC PROPERTIES OF THE TRANSFORM $V$}

In this section we collect the basic properties of the integral transform $V$ defined by

$$
V f(z)=\lambda_{\Omega} \int_{\Omega} f(w) \overline{k_{z}^{2}}(w) d V(w) .
$$

Lemma 1. The operator $Q$ defined by

$$
Q f(z)=\lambda_{\Omega} \int_{\Omega} \frac{K(z, w)^{2}}{K(w, w)} f(w) d V(w)
$$

is a bounded projection from $L^{1}(\Omega, d V)$ onto $L_{a}^{1}(\Omega)$.

Proof. See [9] or use Fubini's theorem.

Proposition 2. If $f \in L^{2}(\Omega, d V)$, then

(1) $P V f=P f$;

(2) $V P f=V f$;

(3) $V^{2} f=V f$. 
Proof. All the equalities follow from Fubini's theorem and Lemma 1 as well as the reproducing property of the Bergman projection $P$. We check (1) for example.

$$
\begin{aligned}
P V f(z) & =\int_{\Omega} K(z, w) V f(w) d V(w) \\
& =\int_{\Omega} K(z, w) d V(w) \lambda_{\Omega} \int_{\Omega} \frac{K(w, u)^{2}}{K(w, w)} f(u) d V(u) \\
& =\lambda_{\Omega} \int_{\Omega} f(u) d V(u) \int_{\Omega} \frac{K(w, u)^{2}}{K(w, w)} K(z, w) d V(w) \\
& =\int_{\Omega} f(u) d V(u) \lambda_{\Omega} \int_{\Omega} \frac{K(u, w)^{2}}{K(w, w)} K(w, z) d V(w) \\
(\text { Lemma } 1) & =\int_{\Omega} f(u) \overline{K(u, z)} d V(u) \\
& =\int_{\Omega} K(z, u) f(u) d V(u)=P f(z) .
\end{aligned}
$$

The other two equalities can be checked similarly.

Proposition 3. $V$ is a bounded operator on $L^{p}(\Omega, d \lambda)$ for all $1 \leq p \leq+\infty$.

Proof. Since each $k_{z}$ is a unit vector in $L_{a}^{2}(\Omega)$, we have

$$
\begin{aligned}
|V f(z)| & \leq \lambda_{\Omega} \int_{\Omega}|f(w)|\left|k_{z}(w)\right|^{2} d V(w) \\
& \leq \lambda_{\Omega}\|f\|_{\infty} \int_{\Omega}\left|k_{z}(w)\right|^{2} d V(w) \\
& =\lambda_{\Omega}\|f\|_{\infty}
\end{aligned}
$$

for all $z$ in $\Omega$. Thus $\|V f\|_{\infty} \leq \lambda_{\Omega}\|f\|_{\infty}$ and $V$ is bounded on $L^{\infty}(d \lambda)$.

If $p \geq 1$, then

$$
|V f(z)|^{p} \leq \lambda_{\Omega}^{p} \int_{\Omega}|f(w)|^{p}\left|k_{z}(w)\right|^{2} d V(w)
$$

since $\left|k_{z}(w)\right|^{2} d V(w)$ is a probability measure for each $z \in \Omega$. Therefore,

$$
\begin{aligned}
\int_{\Omega}|V f(z)|^{p} d \lambda(z) & \leq \lambda_{\Omega}^{p} \int_{\Omega} K(z, z) d V(z) \int_{\Omega}|f(w)|^{p} \frac{|K(z, w)|^{2}}{K(z, z)} d V(w) \\
& =\lambda_{\Omega}^{p} \int_{\Omega}|f(w)|^{p} d V(w) \int_{\Omega}|K(z, w)|^{2} d V(z) \\
& =\lambda_{\Omega}^{p} \int_{\Omega}|f(w)|^{p} K(w, w) d V(w) \\
& =\lambda_{\Omega}^{p} \int_{\Omega}|f(w)|^{p} d \lambda(w) .
\end{aligned}
$$

Proposition 4. $V$. is an orthogonal projection on the Hilbert space $L^{2}(\Omega, d \lambda)$. Proof. Since $V$ is a bounded operator on $L^{2}(d \lambda)$, it suffices to check that $V^{2}=V$ and $V^{*}=V$ on $L^{2}(d \lambda)$, where $V^{*}$ is the adjoint of $V$ on $L^{2}(d \lambda)$. 
$V^{2}=V$ follows from Proposition 2. To prove $V^{*}=V$ on $L^{2}(d \lambda)$, it suffices to prove $\langle V f, g\rangle_{\lambda}=\langle f, V g\rangle_{\lambda}$ for all $f, g$ in $L^{2}(d \lambda)$, where $\langle,\rangle_{\lambda}$ is the (complex) integral pairing with respect to the measure $d \lambda$. Once again, Fubini's theorem gives what we want:

$$
\begin{aligned}
\langle V f, g\rangle_{\lambda} & =\int_{\Omega} \overline{g(z)} d \lambda(z) \lambda_{\Omega} \int_{\Omega} \frac{K(z, w)^{2}}{K(z, z)} f(w) d V(w) \\
& =\lambda_{\Omega} \int_{\Omega} f(w) d V(w) \int_{\Omega} K(z, w)^{2} \overline{g(z)} d V(z) \\
& =\int_{\Omega} f(w) d \lambda(w) \lambda_{\Omega} \int \frac{K(w, z)^{2}}{K(w, w)} g(z) d V(z) \\
& =\int_{\Omega} f(w) \overline{V g(w)} d \lambda(w)=\langle f, V g\rangle_{\lambda} .
\end{aligned}
$$

Remark 1. The boundedness of $V$ on $L^{p}(d \lambda) \quad(1 \leq p \leq+\infty)$ implies that

$$
\langle V f, g\rangle_{\lambda}=\langle f, V g\rangle_{\lambda}
$$

for all $f \in L^{p}(d \lambda), g \in L^{q}(d \lambda)$ with $\frac{1}{p}+\frac{1}{q}=1,1 \leq p \leq \infty$.

Remark 2. Under the usual integral pairing $\langle$,$\rangle (with respect to d V$ ), we have

$$
V^{*} f(z)=\lambda_{\Omega} \int_{\Omega} \frac{K(z, w)^{2}}{K(w, w)} f(w) d V(w)=Q f(z),
$$

where $Q$ is the operator in Lemma 1 .

Proposition 5. For any $\phi \in L^{2}(\Omega, d V)$, we have

(1) $h_{\bar{\phi}}=h_{\overline{P \phi}}$;

(2) $h_{\bar{\phi}}=h_{\overline{V \phi}}$;

(3) $\overline{V \phi(z)}=\lambda_{\Omega}\left\langle h_{\bar{\phi}} k_{z}, \bar{k}_{z}\right\rangle$.

Proof. (1) and (3) follow directly from the definitions of $h_{\bar{\phi}}$ and $V$. (2) is a consequence of (1) and Proposition 2:

$$
h_{\bar{\phi}}=h_{\overline{P \phi}}=h_{\overline{P V \phi}}=h_{\overline{V \phi}} \text {. }
$$

Proposition 6. $V: \mathbb{C}(\bar{\Omega}) \rightarrow \mathbb{C}_{0}(\Omega)$, where $\mathbb{C}(\bar{\Omega})$ is the space of complex functions on $\Omega$ which extend to $\bar{\Omega}$ (the Euclidean closure of $\Omega$ ) continuously.

Proof. We will obtain this in $\S 4$ as a corollary. We give an alternative proof here in the special case $\Omega=B_{n}$. Let $\left\{\phi_{z}: z \in \Omega\right\}$ be the collection of auto- 
morphisms defined in [8], then a change of variable gives

$$
\begin{aligned}
V f(z) & =\lambda_{\Omega} \int_{\Omega} f(w) \overline{k_{z}(w)^{2}} d V(w) \\
& =\lambda_{\Omega} \int_{\Omega} f \circ \phi_{z}(w) \bar{k}_{z}^{2}\left(\phi_{z}(w)\left|k_{z}(w)\right|^{2} d V(w)\right. \\
& =\lambda_{\Omega} \int_{\Omega} f \circ \phi_{z}(w) \frac{\left|k_{z}(w)\right|^{2}}{\overline{k_{z}(w)^{2}}} d V(w) \\
& =\lambda_{\Omega} \int_{\Omega} f \circ \phi_{z}(w) \frac{K(w, z)}{K(z, w)} d V(w) .
\end{aligned}
$$

If $f \in \mathbb{C}(\bar{\Omega})$ and $z_{0} \in \partial \Omega$, then

$$
\lim _{z \rightarrow z_{0}} f \circ \phi_{z}(w)=f\left(z_{0}\right)
$$

for each $w$ since

$$
\lim _{z \rightarrow z_{0}} \phi_{z}(w)=z_{0}
$$

uniformly on compact sets. Therefore, by dominated convergence, we have

$$
\lim _{z \rightarrow z_{0}} V f(z)=\lambda_{\Omega} f\left(z_{0}\right) \int_{\Omega} \frac{K\left(w, z_{0}\right)}{K\left(z_{0}, w\right)} d V(w) .
$$

The reproducing property of $K$ now gives

$$
\begin{aligned}
\int_{\Omega} \frac{K\left(w, z_{0}\right)}{K\left(z_{0}, w\right)} d V(w) & =\lim _{r \rightarrow 1^{-}} \int_{\Omega} \frac{K\left(w, r z_{0}\right)}{K\left(r z_{0}, w\right)} d V(w) \\
& =\lim _{r \rightarrow 1^{-}} \frac{1}{K\left(r z_{0}, r z_{0}\right)}=0 .
\end{aligned}
$$

\section{BOUNDEDNESS OF $h_{\bar{\phi}}$}

Proposition 7. If $\phi \in L^{2}(\Omega, d V)$, then $h_{\bar{\phi}}$ is bounded if and only if $V \phi \in B \mathbb{C}$. In this case, we have

Proof. Since

$$
\left\|h_{\bar{\phi}}\right\| \leq\|V \phi\|_{\infty} \leq \lambda_{\Omega}\left\|h_{\bar{\phi}}\right\|
$$

$$
\overline{V \phi(z)}=\lambda_{\Omega}\left\langle h_{\bar{\phi}} k_{z}, \bar{k}_{z}\right\rangle
$$

and $k_{z}$ is a unit vector for each $z$, we must have $|V \phi(z)| \leq \lambda_{\Omega}\left\|h_{\bar{\phi}}\right\|$ for all $z$, and hence $\|V \phi\|_{\infty} \leq \lambda_{\Omega}\left\|h_{\bar{\phi}}\right\|$. On the other hand, we have by Proposition 5 $\left\|h_{\bar{\phi}}\right\|=\left\|h_{\overline{V \phi}}\right\|$. It is easy to see that $\left\|h_{\psi}\right\| \leq\|\psi\|_{\infty}$ for all $\psi \in L^{\infty}$. Thus we also have $\left\|h_{\bar{\phi}}\right\| \leq\|V \phi\|_{\infty}$.

Theorem 8 [10]. If $\phi$ is holomorphic and in $L^{2}(\Omega, d V)$, then the following are equivalent:

(1) $h_{\bar{\phi}}$ is bounded; 
(2) $V \phi \in B \mathbb{C}(\Omega)$;

(3) $\phi \in P L^{\infty}(\Omega)$;

(4) $\left|\int_{\Omega} f(z) \overline{\phi(z)} d V(z)\right| \leq C \int_{\Omega}|f(z)| d V(z)$ for some constant $C>0$ and all $f \in H^{\infty}(\Omega)$.

Moreover, the following quantities are equivalent: $\left\|h_{\bar{\phi}}\right\|,\|V \phi\|_{\infty}, \inf \left\{\|\psi\|_{\infty}\right.$ : $\phi=P \psi\}, \sup \left\{\left|\int_{\Omega} f(z) \overline{\phi(z)} d V(z)\right|:\|f\|_{1} \leq 1, f \in H^{\infty}\right\}$, where $\|f\|_{1}=$ $\int_{\Omega}|f(z)| d V(z)$.

Proof. The equivalence of (1) and (2) was proved in Proposition 7 along with the inequalities $\left\|h_{\bar{\phi}}\right\| \leq\|V \phi\|_{\infty} \leq \lambda_{\Omega}\left\|h_{\bar{\phi}}\right\|$.

By Proposition 2, $\phi \in L^{2}(\Omega, d V)$ is holomorphic implies that $\phi=P V \phi$. Therefore $\inf \left\{\|\psi\|_{\infty}: \phi=P \psi\right\} \leq\|V \phi\|_{\infty}$.

If $\phi=P \psi$ with $\psi \in L^{\infty}$, then $\left\|h_{\bar{\phi}}\right\|=\left\|h_{\bar{P} \psi}\right\|=\left\|h_{\bar{\psi}}\right\| \leq\|\psi\|_{\infty}$. This implies that $\left\|h_{\bar{\phi}}\right\| \leq \inf \left\{\|\psi\|_{\infty}: \phi=P \psi\right\}$.

It is well-known [2] that for each $z \in \Omega$, the function $k_{z}(w)$ is holomorphic in a neighbourhood of $\Omega$. In particular, $k_{z} \in H^{\infty}$ for all $z \in \Omega$. Thus

$$
\begin{aligned}
\|V \phi\|_{\infty} & =\sup \{|V \phi(z)|: z \in \Omega\} \\
& =\lambda_{\Omega} \sup \left\{\left|\int_{\Omega} \phi(w) \overline{k_{z}^{2}(w)} d V(w)\right|: z \in \Omega\right\} \\
& \leq \lambda_{\Omega} \sup \left\{\left|\int_{\Omega} \phi(w) \overline{f(w)} d V(w)\right|:\|f\|_{1} \leq 1, f \in H^{\infty}\right\} .
\end{aligned}
$$

Finally, if $f \in H^{\infty}$ and $\|f\|_{1} \leq 1$, then

$$
\begin{aligned}
\int_{\omega} f(z) \overline{\phi(z)} d V(z) & =\int_{\Omega} Q f(z) \overline{\phi(z)} d V(z) \\
& =\int_{\Omega} f(z) \overline{V \phi(z)} d V(z)
\end{aligned}
$$

by Remark 2 in the last section. It follows that

$$
\left|\int_{\Omega} f(z) \overline{\phi(z)} d V(z)\right| \leq\|V \phi\|_{\infty}\|f\|_{1} \leq\|V \phi\|_{\infty},
$$

completing the proof of Theorem 8 .

Corollary 9. Let $X=P L^{\infty}(\Omega)$ be equipped with the norm $\|\phi\|_{*}=\left\|h_{\bar{\phi}}\right\|$, then $L_{a}^{1}(\Omega)^{*}=X$ with equivalent norms.

Proof. Suppose $\xi \in L_{a}^{1}(\Omega)^{*}$, then the Hahn-Banach extension theorem implies that $\xi$ can be extended to $L^{1}(\Omega, d V)$ without increasing the norm. Since $L^{1}(\Omega, d V)^{*}=L^{\infty}(\Omega)$, there must exist a function $\psi \in L^{\infty}(\Omega)$ such that 
$\|\xi\|=\|\psi\|_{\infty}$ and $\xi(f)=\int_{\Omega} f(z) \overline{\psi(z)} d V(z)$ for all $f \in L_{a}^{1}(\Omega)$. Let $\phi=P \psi \in$ $X$, then for all $f \in H^{\infty}$ (dense in $L_{a}^{1}(\Omega)$ ), we have

$$
\begin{aligned}
\xi(f) & =\int_{\Omega} f(z) \overline{\psi(z)} d V(z) \\
& =\int_{\Omega} P f(z) \overline{\psi(z)} d V(z) \\
& =\int_{\Omega} f(z) \overline{P \psi(z)} d V(z) \\
& =\int_{\Omega} f(z) \overline{\phi(z)} d V(z) .
\end{aligned}
$$

Moreover,

$$
\|\phi\|_{*} \leq \sup \left\{\left|\int_{\Omega} f(z) \overline{\phi(z)} d V(z)\right|: f \in H^{\infty},\|f\|_{1} \leq 1\right\}=\|\xi\| .
$$

Conversely, if $\phi \in X$, then $\phi$ induces a bounded linear functional $\xi_{\phi}$ on $L_{a}^{1}(\Omega)$ as follows:

$$
\zeta_{\phi}(f)=\int_{\Omega} f(z) \overline{\phi(z)} d V(z), \quad f \in H^{\infty}\left(\text { dense in } L_{a}^{1}(\Omega)\right) .
$$

Moreover, we have

$$
\left\|\xi_{\phi}\right\|=\sup \left\{\left|\int_{\Omega} f(z) \overline{\phi(z)} d V(z)\right|: f \in H^{\infty},\|f\|_{1} \leq 1\right\} \leq \lambda_{\Omega}\|\phi\|_{*} .
$$

Remark. Since the dual of any Banach space is still a Banach space, thus $X$ is complete in the norm \|\|$_{*}$. Another way of seeing this is to consider $X$ as a quotient space:

$$
P: L^{\infty}(\Omega) \rightarrow X .
$$

\|\|$_{*}$ is equivalent to the quotient norm. The quotient space of any Banach space is still a Banach space.

\section{CoMpactNess of $h_{\bar{\phi}}$}

Proposition 10. Suppose $\phi \in L^{2}(\Omega, d V)$ with $V \phi \in B \mathbb{C}$, then $h_{\bar{\phi}}$ is compact if and only if $V \phi \in \mathbb{C}_{0}$ if and only if $V \phi \in \mathbb{C}(\bar{\Omega})$.

Proof. Since $\overline{V \phi(z)}=\lambda_{\Omega}\left\langle h_{\bar{\phi}}, k_{z}, \bar{k}_{z}\right\rangle$ and $k_{z} \rightarrow 0$ weakly as $z \rightarrow \partial \Omega$, thus $h_{\bar{\phi}}$ is compact implies that $V \phi \in \mathbb{C}_{0}$ which in turn implies that $V \phi \in \mathbb{C}(\bar{\Omega})$.

On the other hand, if $V \phi \in \mathbb{C}(\bar{\Omega})$, then $V \phi$ can be approximated in $L^{\infty}(\Omega)$ by finite linear combinations of $z^{\alpha} \bar{z}^{\beta}$ (Stone-Weierstrass). Since each $h_{\bar{z}^{\alpha} z^{\beta}}$ is a finite rank operator and $\psi \mapsto h_{\psi}$ is norm continuous, $h_{\overline{V \phi}}$ can be approximated in norm by finite linear combinations of finite rank operators. Hence $h_{\bar{\phi}}=h_{\overline{V_{\phi}}}$ is compact.

Corollary 11. $V: \mathbb{C}(\bar{\Omega}) \rightarrow \mathbb{C}_{0}(\Omega)$.

Proof. Suppose $\phi \in \mathbb{C}(\bar{\Omega})$, then $h_{\bar{\phi}}$ is compact by the proof of Proposition 10 . Thus $V \in \mathbb{C}_{0}(\Omega)$ by the proposition. 
Theorem 12 [10]. If $\phi$ is holomorphic in $\Omega$ with $h_{\bar{\phi}}$ bounded, then the following are equivalent:

(1) $h_{\bar{\phi}}$ is compact;

(2) $V \phi \in \mathbb{C}_{0}(\Omega)$;

(3) $V \phi \in \mathbb{C}(\bar{\Omega})$;

(4) $\phi \in P \mathbb{C}_{0}(\Omega)$;

(5) $\phi \in P \mathbb{C}(\bar{\Omega})$.

Proof. The equivalences of (1), (2), and (3) are in Proposition 10. $\phi=P V \phi$ gives the implication (2) $\Rightarrow(4)$. (4) $\Rightarrow(5)$ is trivial. If $\phi=P \psi$ with $\psi \in$ $\mathbb{C}(\bar{\Omega})$, then $h_{\bar{\phi}}=h_{\bar{\psi}}$ is compact by the Stone-Weierstrass argument used in the proof of Proposition 10, completing the proof of Theorem 12.

Recall that $X=P L^{\infty}(\Omega)$ is the Banach space with norm $\|f\|_{*}=\left\|h_{\bar{f}}\right\|$. Let $X_{0}$ be the closed subspace of $X$ consisting of $f$ such that $h_{\bar{f}}$ is compact. It is easy to see that the polynomials are dense in $X_{0}$ [10], thus $X_{0}$ is separable ( $X$ is not!).

Corollary 13. $X_{0}^{*}=L_{a}^{1}(\Omega)$ with equivalent norms.

Proof. Suppose $f \in L_{a}^{1}(\Omega)$, then $f$ induces a bounded linear functional $\xi_{f}$ on $X$ :

$$
\xi_{f}(g)=\int_{\Omega} g(z) \overline{f(z)} d V(z), \quad g \in \mathscr{P},
$$

where $\mathscr{P}$ is the set of polynomials (dense in $X_{0}$ ). By Corollary $9,\left\|\xi_{f}\right\| \leq$ $\lambda_{\Omega}\|f\|_{1}$.

Conversely, suppose that $\xi \in X_{0}^{*}$, we wish to find a function $f \in L_{a}^{1}(\Omega)$ such that $\xi=\xi_{f}$. Consider the mapping

$$
V: X_{0} \rightarrow \mathbb{C}_{0}(\Omega) \text {. }
$$

By Proposition 7, $V$ is an imbeding, thus the image $V X_{0}$ is a closed subspace of $\mathbb{C}_{0}(\Omega)$. Moreover,

$$
\xi \circ V^{-1}: V X_{0} \rightarrow \mathbb{C}
$$

is a bounded linear functional on $V X_{0}$ with norm $\leq\left\|V^{-1}\right\|\|\xi\|$. Extend $\xi \circ V^{-1}$ to the space $\mathbb{C}_{0}(\Omega)$ by the Hahn-Banach theorem. The Riesz representation theorem then produces a finite complex Borel measure $\mu$ on $\Omega$ such that

$$
\xi \circ V^{-1}(f)=\int_{\Omega} f(z) d \mu(z)
$$

for all $f$ in $V X_{0}$. It follows that

$$
\xi(g)=\int_{\Omega} V g(z) d \mu(z)
$$


for all $g$ in $X_{0}$. If $g \in X_{0}$ is a polynomial, then Fubini's theorem gives

$$
\begin{aligned}
\xi(g) & =\int_{\Omega} d \mu(z) \lambda_{\Omega} \int_{\Omega} \frac{K(z, w)^{2}}{K(z, z)} g(w) d V(w) \\
& =\int_{\Omega} g(w) d V(w) \lambda_{\Omega} \int_{\Omega} \frac{K(z, w)^{2}}{K(z, z)} d \mu(z) .
\end{aligned}
$$

Let

$$
f(w)=\lambda_{\Omega} \int_{\Omega} \frac{K(w, z)^{2}}{K(z, z)} d \bar{\mu}(z),
$$

then $f \in L_{a}^{1}(\Omega)$ with

$$
\begin{aligned}
\|f\|_{1} & \leq \lambda_{\Omega} \int_{\Omega} d V(w) \int_{\Omega} \frac{|K(w, z)|^{2}}{K(z, z)} d|\mu|(z) \\
& =\lambda_{\Omega} \int_{\Omega} d|\mu|(z) \int_{\Omega} \frac{|K(w, z)|^{2}}{K(z, z)} d V(w) \\
& =\lambda_{\Omega} \int d|\mu|(z)=\lambda_{\Omega}\|\mu\| \leq \lambda_{\Omega}\|\xi\|\left\|V^{-1}\right\| .
\end{aligned}
$$

Moreover, we have

$$
\xi(g)=\int_{\Omega} g(w) \overline{f(w)} d V(w)=\xi_{f}(g)
$$

for all $g$ in $\mathscr{P}$ (dense in $X_{0}$ ). This completes the proof of Corollary 13 .

Remark. The results in this section as well as the last section are mostly taken from [10]. The approach here is improved.

\section{Schatten-Von Neumann class Hankel operators}

Suppose $A$ is a bounded operator from $L_{a}^{2}(\Omega)$ into $\overline{L_{a}^{2}(\Omega)}$, then $A^{*} A$ is a positive operator on the Hilbert space $L_{a}^{2}(\Omega)$. We say that $A$ is in the Schatten-Von Neumann $p$-ideal $S_{p}$ if $\operatorname{tr}\left(\left(A^{*} A\right)^{p / 2}\right]$ is finite. We will be mainly concerned with the case $1 \leq p<\infty$. In this case, each $S_{p}$ is a Banach space. We will write $S_{\infty}$ for the set of all bounded operators. Note that some authors write $S_{\infty}$ for the set of all compact operators. [5] contains a fair amount of information about the ideals $S_{p}$. We will need the interpolating property:

$$
\left[S_{p_{0}}, S_{p_{1}}\right]_{\theta}=S_{p}
$$

if $1 \leq p_{0}<p_{1} \leq+\infty$ and $1 / p=(1-\theta) / p_{0}+\theta / p_{1}$ for $\theta \in(0,1)$, where $[,]_{\theta}$ is the functor of complex interpolation. The $L^{p}$-spaces also have the same interpolating properties:

$$
\left[L^{p_{0}}(d \mu), L^{p_{1}}(d \mu)\right]_{\theta}=L^{p}(d \mu)
$$

if $1 \leq p_{0}<p_{1} \leq+\infty$ and $1 / p=(1-\theta) / p_{0}+\theta / p_{1}$ with $\theta \in(0,1)$. Any book on interpolation of Banach spaces should contain the above results. 
Lemma 14. For $1 \leq p \leq+\infty$, we have

$$
\phi \in L^{p}(d \lambda) \Rightarrow h_{\phi} \in S_{p} .
$$

Proof. The case $p=+\infty$ is clear. The case $p=2$ is also easy. Recall that

$$
h_{\phi} f(z)=\bar{P}(\phi f)(z)=\int_{\Omega} K(w, z) \phi(w) f(w) d V(w) .
$$

Thus $h_{\phi}$ is an integral operator with kernel $K(w, z) \phi(w)$ satisfying

$$
\begin{aligned}
\int_{\Omega} \int_{\Omega}|K(w, z) \phi(w)|^{2} d V(z) d V(w) \\
\quad=\int_{\Omega}|\phi(w)|^{2} d V(w) \int_{\Omega}|K(z, w)|^{2} d V(z) \\
\quad=\int_{\Omega}|\phi(w)|^{2} K(w, w) d V(w) \\
\quad=\|\phi\|_{L^{2}(d \lambda)}^{2} .
\end{aligned}
$$

Therefore, $\phi \in L^{2}(d \lambda)$ implies $h_{\phi} \in S_{2}$. By complex interpolation, we have $\phi \in L^{p}(d \lambda)$ implies that $h_{\phi} \in S_{p}$ for all $2<p<+\infty$. The case $1 \leq p \leq 2$ is a little more difficult. Again we will use complex interpolation. It suffices to check the endpoint $p=1$. The method below is contained in [6].

First observe that

$$
h_{\phi}=\int_{\Omega} \phi(w) h_{\bar{K}_{w}} d V(w),
$$

where $K_{w}(z)=K(z, w)$. In fact, if $f \in L_{a}^{2}(\Omega)$, then

$$
\begin{aligned}
h_{\bar{K}_{w}} f(z) & =\bar{P}\left(\bar{K}_{w} f\right)(z) \\
& =\int_{\Omega} K(u, z) \bar{K}_{w}(u) f(u) d V(u) \\
& =\int_{\Omega} K(u, z) K(w, u) f(u) d V(u) \\
& =K(w, z) f(w)=f(w) \bar{K}_{w}(z)
\end{aligned}
$$

and

$$
\begin{aligned}
\int_{\Omega} \phi(w) h_{\bar{K}_{w}} f(z) d V(w) & =\int_{\Omega} \phi(w) f(w) \bar{K}_{w}(z) d V(w) \\
& =\int_{\Omega} K(w, z) \phi(w) f(w) d V(w) \\
& =h_{\phi} f(z) .
\end{aligned}
$$

Therefore, we have

$$
h_{\phi}=\int_{\Omega} \phi(w) h_{\bar{K}_{w}} d V(w)
$$

with each $h_{\bar{K}_{w}}$ being a rank 1 operator. 
Suppose $\phi \in L^{1}(\Omega, d \lambda)$, we wish to show that $h_{\phi} \in S_{1}$. It suffices to show that the above integral converges in $S_{1}$ (because $S_{1}$ is complete). Since $h_{\bar{K}_{w}}$ is a rank 1 operator, we have

$$
\left\|h_{\bar{K}_{w}}\right\|_{S_{1}}=\left\|h_{\bar{K}_{w}}\right\| .
$$

Note that

$$
h_{\bar{K}_{w}} f=f(w) \bar{K}_{w},
$$

thus

$$
\begin{aligned}
\left\|h_{\bar{K}_{w}}\right\| & =\sup \left\{|f(w)|\left\|\bar{K}_{w}\right\|_{2}:\|f\|_{2}=1 \text { in } L_{a}^{2}(\Omega)\right\} \\
& =\sqrt{K(w, w)} \sup \left\{|f(w)|:\|f\|_{2}=1 \text { in } L_{a}^{2}(\Omega)\right\} \\
& =K(w, w) .
\end{aligned}
$$

Therefore

$$
\begin{aligned}
\int_{\Omega}\left\|\phi(w) h_{\bar{K}_{w}}\right\|_{S_{1}} d V(w) & =\int_{\Omega}|\phi(w)| K(w, w) d V(w) \\
& =\int_{\Omega}|\phi(w)| d \lambda(w)<+\infty
\end{aligned}
$$

if $\phi \in L^{1}(d \lambda)$. This completes the proof of Lemma 14 .

Theorem 15. Suppose $1 \leq p \leq \infty$ and $V \phi \in B \mathbb{C}$, then

$$
h_{\bar{\phi}} \in S_{p} \Leftrightarrow V \phi \in L^{p}(d \lambda) \text {. }
$$

Proof. The case $p=+\infty$ was settled in $\S 3$. Since $h_{\bar{\phi}}=h_{\overline{V \phi}}$, Lemma 14 gives the implication $V \phi \in L^{p}(d \lambda) \Rightarrow h_{\bar{\phi}} \in S_{p}$.

Suppose $h_{\bar{\phi}} \in S_{p}$, then $\left(h_{\bar{\phi}}^{*} h_{\bar{\phi}}\right)^{p / 2} \in S_{1}$. By [1] or [11],

$$
\operatorname{tr}\left[\left(h_{\bar{\phi}}^{*} h_{\bar{\phi}}\right)^{p / 2}\right]=\int_{\Omega}\left\langle\left(h_{\bar{\phi}}^{*} h_{\bar{\phi}}\right)^{p / 2} k_{z}, k_{z}\right\rangle K(z, z) d V(z) .
$$

If $p \geq 2$, then the generalized Hölder inequality in [1] gives

$$
\begin{aligned}
\operatorname{tr}\left[\left(h_{\bar{\phi}}^{*} h_{\bar{\phi}}\right)^{p / 2}\right] & \geq \int_{\Omega}\left\langle h_{\bar{\phi}}^{*} h_{\bar{\phi}} k_{z}, k_{z}\right\rangle^{p / 2} K(z, z) d V(z) \\
& =\int_{\Omega}\left\|h_{\bar{\phi}} k_{z}\right\|^{p} K(z, z) d V(z) .
\end{aligned}
$$

Since $k_{z}$ is a unit vector, the Cauchy-Schwarz inequality gives

$$
\left\|h_{\bar{\phi}} k_{z}\right\| \geq\left|\left\langle h_{\bar{\phi}} k_{z}, \bar{k}_{z}\right\rangle\right|=\lambda_{\Omega}^{-1}|V \phi(z)| \text {. }
$$

Thus we get

$$
\lambda_{\Omega} \operatorname{tr}\left[\left(h_{\bar{\phi}}^{*} h_{\bar{\phi}}\right)^{p / 2}\right] \geq \int_{\Omega}|V \phi(z)|^{p} d \lambda(z)
$$

for all $p \geq 2$. This gives the implication $h_{\bar{\phi}} \in S_{p} \Rightarrow V \phi \in L^{p}(d \lambda)$ for all $p \geq 2$. 
The case $1 \leq p \leq 2$ is once again more difficult. We outline a method (used in [7]) to prove the implication $h_{\bar{\phi}} \in S_{p} \Rightarrow V f \in L^{p}(d \lambda)$ for all $1 \leq p<+\infty$. (At least for the case of the open unit ball or the poly-disc in $\mathbb{C}^{n}$-added in proof.)

Fix a sequence of points $\left\{a_{n}\right\}$ in $\Omega$ such that

(1) $\Omega=\bigcup_{n=1}^{\infty} E\left(a_{n}\right)$, where $E\left(a_{n}\right)$ is the Bergman metric ball with center $a_{n}$ and radius $r$ (some fixed positive number);

(2) There exists a constant $C>0$ such that every function $f$ in $L_{a}^{2}(\Omega)$ can be written as

$$
f(z)=\sum_{n=1}^{\infty} \lambda_{n} k_{a_{n}}(z)
$$

with

$$
\|f\|_{2} \leq C \text { inf }\left\{\sqrt{\sum_{n=1}^{\infty}\left|\lambda_{n}\right|^{2}}: f=\sum_{n=1}^{\infty} \lambda_{n} k_{a_{n}}\right\} .
$$

For the construction of such a sequence $\left\{a_{n}\right\}$, see [4].

Define an operator $A$ on $L_{a}^{2}(\Omega)$ by letting

$$
A e_{n}=k_{a_{n}}, \quad n=1,2, \ldots,
$$

where $\left\{e_{n}\right\}$ is a fixed orthonormal basis of $L_{a}^{2}(\Omega)$. If $f \in L_{a}^{2}(\Omega)$ with $f=$ $\sum_{n=1}^{\infty} f_{n} e_{n}$, then

$$
A f=\sum_{n=1}^{\infty} f_{n} k_{a_{n}}
$$

and by (2) above,

$$
\begin{aligned}
\|A f\| & \leq C \inf \left\{\sqrt{\sum_{n=1}^{\infty}\left|\lambda_{n}\right|^{2}}: A f=\sum_{n=1}^{\infty} \lambda_{n} k_{a_{n}}\right\} \\
& \leq C \sqrt{\sum_{n=1}^{\infty}\left|f_{n}\right|^{2}}=C\|f\| .
\end{aligned}
$$

Thus $A$ is a bounded linear operator. Let $\bar{A}$ be the operator on $\overline{L_{a}^{2}(\Omega)}$ defined by $\bar{A} \bar{e}_{n}=\bar{k}_{a_{n}}$, then $\bar{A}$ is also bounded.

Suppose $h_{\bar{\phi}} \in S_{p}$ with $p \geq 1$, then we also have $\bar{A}^{*} h_{\bar{\phi}} A \in S_{p}$. This implies that

$$
\sum_{n=1}^{\infty}\left|\left\langle\bar{A}^{*} h_{\bar{\phi}} A e_{n}, \bar{e}_{n}\right\rangle\right|^{p}<+\infty,
$$

or

$$
\sum_{n=1}^{\infty}\left|\left\langle h_{\bar{\phi}} k_{a_{n}}, \bar{k}_{a_{n}}\right\rangle\right|^{p}<+\infty,
$$


or

$$
\sum_{n=1}^{\infty}\left|V \phi\left(a_{n}\right)\right|^{p}<+\infty .
$$

It is not difficult to show that [11] $V \phi(z)$ behaves like $V \phi\left(a_{n}\right)$ for $z \in E\left(a_{n}\right)$. Also [11] $K(z, z)$ behaves like $K\left(a_{n}, a_{n}\right) \cong \frac{1}{E\left(a_{n}\right)}$ for $z$ in $E\left(a_{n}\right)$. It follows that

$$
\begin{aligned}
\int_{\Omega}|V \phi(z)|^{p} d \lambda(z) & \leq \sum_{n=1}^{\infty} \int_{E\left(a_{n}\right)}|V \phi(z)|^{p} K(z, z) d V(z) \\
& \leq C_{1} \sum_{n=1}^{\infty} \frac{1}{\left|E\left(a_{n}\right)\right|} \int_{E\left(a_{n}\right)}|V \phi(z)|^{p} d V(z) \\
& \leq C_{2} \sum_{n=1}^{\infty} \frac{1}{\left|E\left(a_{n}\right)\right|} \int_{E\left(a_{n}\right)}\left|V \phi\left(a_{n}\right)\right|^{p} d V(z) \\
& =C_{2} \sum_{n=1}^{\infty}\left|V \phi\left(a_{n}\right)\right|^{p}<+\infty,
\end{aligned}
$$

completing the proof of Theorem 15.

Corollary 16. Let $\left\{a_{n}\right\}$ be as in the proof of the above theorem, then for all $\phi \in L^{2}(\Omega, d V)$ we have

(1) $h_{\bar{\phi}}$ is bounded $\Leftrightarrow\left\{V \phi\left(a_{n}\right)\right\} \in l^{\infty}$;

(2) $h_{\bar{\phi}}$ is compact $\Leftrightarrow\left\{V \phi\left(a_{n}\right)\right\} \in c_{0}$;

(3) $h_{\bar{\phi}}$ is in $S_{p} \Leftrightarrow\left\{V \phi\left(a_{n}\right)\right\} \in l^{p},(p \geq 1)$.

Corollary 17. If $\phi$ is holomorphic in $\Omega$ with $V \phi \in B \mathbb{C}$, then for all $p \geq 1$, we have $h_{\bar{\phi}} \in S_{p}$ if and only if $\phi \in P L^{p}(d \lambda)$.

Proof. If $\phi=P \psi$ with $\psi \in L^{p}(d \lambda)$, then

$$
h_{\bar{\phi}}=h_{\overline{P \psi}}=h_{\bar{\psi}} \in S_{p}
$$

by Lemma 14. Conversely, if $h_{\bar{\phi}} \in S_{p}$, then $V \phi \in L^{p}(d \lambda)$ by Theorem 15 . It follows that $\phi=P V \phi$ is in $P L^{p}(d \lambda)$.

\section{THE OPEN UNIT BALL $B_{n}$}

In this section, we specialize to the special case $\Omega=B_{n}$, the open unit ball in $\mathbb{C}^{n}$. We show that if $f$ is holomorphic in $B_{n}$, then $V f$ behaves like the following functions:

$$
\left(1-|z|^{2}\right)^{n+1} \frac{\partial^{n+1} f}{\partial z^{\alpha}}(z) \quad(|\alpha|=n+1) .
$$

To see why this is true, we first observe that for $f \in L_{a}^{2}(\Omega)$,

$$
f(z)=\int_{B_{n}} \frac{f(w) d V(w)}{(1-\langle z, w\rangle)^{n+1}},
$$




$$
\left(1-|z|^{2}\right)^{n+1} \frac{\partial^{n+1} f}{\partial z^{\alpha}}(z)=\frac{(2 n+1) !}{n !} \int_{B_{n}} \frac{\left(1-|z|^{2}\right)^{n+1} \bar{w}^{\alpha} f(w)}{(1-\langle z, w\rangle)^{2 n+2}} .
$$

On the other hand,

$$
V f(z)=\lambda_{B_{n}} \int_{B_{n}} \frac{\left(1-|z|^{2}\right)^{n+1} f(w)}{(1-\langle z, w\rangle)^{2 n+2}} d V(w) .
$$

Therefore, the only difference between $V f(z)$ and $\left(1-|z|^{2}\right)^{n+1} \partial^{n+1} f(z) / \partial z^{\alpha}$ $(|\alpha|=n+1)$ is the factor $\bar{w}^{\alpha}$ in the integrand. It suggests that $V f(z)$ should behave like the derivatives $(1-|z|)^{n+1} \partial^{n+1} f(z) / \partial z^{\alpha} \quad(|\alpha|=n+1)$. The following theorem shows that this is indeed the case. Let

$$
T_{\alpha} f(z)=\left(1-|z|^{2}\right)^{n+1} \frac{\partial^{n+1} f}{\partial z^{\alpha}}(z) \quad(|\alpha|=n+1) .
$$

Theorem 18. If $f \in L_{a}^{2}\left(B_{n}\right.$ and $p \geq 1$, then

(1) $V f \in B \mathbb{C} \Leftrightarrow T_{\alpha} f \in B \mathbb{C}$ for all $|\alpha|=n+1$;

(2) $V f \in \mathbb{C}_{0} \Leftrightarrow T_{\alpha} f \in \mathbb{C}_{0}$ for all $|\alpha|=n+1$;

(3) $V f \in L^{p}(d \lambda) \Leftrightarrow T_{\alpha} f \in L^{p}(d \lambda)$ for all $|\alpha|=n+1$.

Remark. Since $V f \in B \mathbb{C}$ iff $f \in P L^{\infty}(\Omega)$ and $V f \in \mathbb{C}_{0}$ iff $f \in P \mathbb{C}_{0}(\Omega)$, (1) and (2) above follow from the results in [12]. Our idea of proving (3) above is still the same as that in [12]. Although Theorem 18 is conceivably obvious in view of the observation proceeding it, we still need some technical lemmas to make the proof complete.

Recall that the fundamental theorem of calculus gives

$$
f(z)-f(0)=\sum_{i=1}^{n} z_{i} \int_{0}^{1} \frac{\partial f}{\partial z_{i}}(t z) d t .
$$

Let

$$
A_{i} f(z)=\int_{0}^{1} \frac{\partial f}{\partial z_{i}}(t z) d t, \quad 1 \leq i \leq n .
$$

For any $n$-tuple $\alpha=\left(\alpha_{1}, \ldots, \alpha_{n}\right)$ of nonnegative integers, let

$$
A_{\alpha}=A_{1}^{\alpha_{1}} \cdots A_{n}^{\alpha_{n}},
$$

acting on holomorphic functions (the $A_{i}$ 's commute with each other).

Lemma 19. For all $\sigma \leq 0$ and $p \geq 1$, the operators $A_{\alpha}$ are bounded on $L_{a}^{p}\left(B_{n}, K(z, z)^{\sigma} d V(z)\right)$.

This is basically a Gleason problem [8]. It can either be proved by the method in [12], or be proved by using Cauchy integrals. Either way, it suffices to consider the operators $A_{1}, \ldots, A_{n}$. The boundedness of $A_{\alpha}$ follows from the boundedness of $A_{1}, \ldots, A_{n}$. We omit the details here. 
Corollary 20. There exists a constant $\mathrm{C}>0$ such that for all holomorphic $f$ with $\partial^{|\alpha|} f(0) / \partial z^{\alpha}=0$ (for all $\left.|\alpha| \leq n\right)$ we have

$$
f(z)=\sum_{|\alpha|=n+1} z^{\alpha} A_{\alpha} f(z) .
$$

Moreover, $\left\|K^{-1} A_{\alpha} f\right\|_{L^{p}(d \lambda)} \leq \mathrm{C}\left\|K^{-1} f\right\|_{L^{p}(d \lambda)}$ for all $|\alpha|=n+1$, where $K^{-1} f(z)=K(z, z)^{-1} f(z)=\left(1-|z|^{2}\right)^{n+1} f(z)$.

We can now prove Theorem 18 . Only (3) needs to be checked.

Suppose $V f \in L^{p}(d \lambda)$. The equality $f=P V f$ gives

$$
f(z)=\int_{B_{n}} \frac{V f(w)}{(1-\langle z, w\rangle)^{n+1}} d V(w) .
$$

Differentiating under the integral sign leads to

$$
\left(1-|z|^{2}\right)^{n+1} \frac{\partial^{n+1} f}{\partial z^{\alpha}}(z)=\frac{(2 n+1) !}{n !} \int_{B_{n}} \frac{\left(1-|z|^{2}\right)^{n+1} \bar{w}^{\alpha} V f(w)}{(1-\langle z, w\rangle)^{2 n+2}} d V(w) .
$$

Let $M_{\alpha}$ be the operator defined by

$$
M_{\alpha} f(z)=\frac{(2 n+1) !}{n ! \lambda_{B_{n}}} \bar{z}^{\alpha} f(z)=(n+1) ! \bar{z}^{\alpha} f(z),
$$

then the above equality becomes

$$
T_{\alpha} f=V M_{\alpha} V f .
$$

Clearly, $M_{\alpha}$ is bounded on $L^{p}(d \lambda)$. Also by Proposition $3, V$ is bounded on $L^{p}(d \lambda)$. Thus $V f \in L^{p}(d \lambda)$ implies that $T_{\alpha} f=V M_{\alpha}(V f) \in L^{p}(d \lambda)$ for all $|\alpha|=n+1$.

Conversely, suppose $T_{\alpha} f(z) \in L^{p}(d \lambda)$ for all $|\alpha|=n+1$. We wish to show that $V f \in L^{p}(d \lambda) \quad(p \geq 1)$. Since $V g \in L^{p}(d \lambda)$ for all polynomials $g$. We may assume that $\partial^{|\alpha|} f(0) / \partial z^{\alpha}=0$ for all $|\alpha| \leq n$. Thus

$$
f(z)=\sum_{|\alpha|=n+1} z^{\alpha} f_{\alpha}(z)
$$

where $f_{\alpha}(z)=A_{\alpha} f(z)$. In order to prove $V f \in L^{p}(d \lambda)$, it suffices to show that there exists a constant $C>0$ such that

$$
\left|\langle V f, g\rangle_{\lambda}\right| \leq C\|g\|_{L^{q}(d \lambda)}
$$

for all $g \in L^{q}(d \lambda)$ with $1 / p+1 / q=1$. By the proof of Proposition 4,

$$
\langle V f, g\rangle_{\lambda}=\langle f, V g\rangle_{\lambda}=\langle V f, V g\rangle_{\lambda} \text {. }
$$

Write $V g(z)=K(z, z)^{-1} \tilde{g}(z)$ with $\widetilde{g}$ holomorphic. It suffices to produce a constant $C>0$ such that

$$
\left|\left\langle V f, K^{-1} \tilde{g}\right\rangle_{\lambda}\right| \leq C\left\|K^{-1} \tilde{g}\right\|_{L^{q}(d \lambda)} .
$$


Since $\partial^{|\alpha|} f(0) / \partial z^{\alpha}=0 \quad(|\alpha| \leq n), K(z, z)^{-1}=\left(1-|z|^{2}\right)^{n+1}$ is unitarily invariant, we must have

$$
\left\langle f, K^{-1} \tilde{g}\right\rangle_{\lambda}=\left\langle f, K^{-1} \tilde{g}_{1}\right\rangle_{\lambda}
$$

where $\tilde{g}(z)=\widetilde{g}_{0}(z)+\widetilde{g}_{1}(z)$ with

$$
\widetilde{g}_{1}(z)=\sum_{|\alpha|=n+1} z^{\alpha} A_{\alpha} \widetilde{g}(z) .
$$

Let $\widetilde{g}_{\alpha}=A_{\alpha} \widetilde{g}$, then

$$
\begin{aligned}
\left\langle f, K^{-1} \widetilde{g}_{1}\right\rangle_{\lambda} & =\sum_{|\alpha|=n+1} \int_{\Omega} f(z) \bar{z}^{\alpha} \overline{\widetilde{g}}_{\alpha}(z) d V(z) \\
& =\frac{1}{(n+1) !} \sum_{|\alpha|=n+1}\left\langle f, M_{\alpha}^{*} g_{\alpha}\right\rangle \\
& =\frac{1}{(n+1) !} \sum_{|\alpha|=n+1}\left\langle M_{\alpha} f, \widetilde{g}_{\alpha}\right\rangle
\end{aligned}
$$

where $\langle$,$\rangle is the usual pairing with respect to d V$ and $M_{\alpha}^{*} f(z)=z^{\alpha} f(z)$. Going back to the pairing $\langle,\rangle_{\lambda}$, we get

$$
\left\langle f, K^{-1} \tilde{g}\right\rangle_{\lambda}=\frac{1}{(n+1) !} \sum_{|\alpha|=n+1}\left\langle M_{\alpha} f, K^{-1} \widetilde{g}_{\alpha}\right\rangle_{\lambda}
$$

Since $K^{-1} \widetilde{g}$ is in the image of $V$ and $V$ is a projection in the pairing $\langle,\rangle_{\lambda}$, we have

$$
\begin{aligned}
\left\langle f, K^{-1} \tilde{g}\right\rangle_{\lambda} & =\frac{1}{(n+1) !} \sum_{|\alpha|=n+1}\left\langle V M_{\alpha} f, K^{-1} \widetilde{g}_{\alpha}\right\rangle_{\lambda} \\
& =\frac{1}{(n+1) !} \sum_{|\alpha|=n+1}\left\langle T_{\alpha} f, K^{-1} \widetilde{g}_{\alpha}\right\rangle_{\lambda} .
\end{aligned}
$$

It follows that

$$
\left|\left\langle f, K^{-1} \widetilde{g}\right\rangle_{\lambda}\right| \leq \frac{1}{(n+1) !} \sum_{|\alpha|=n+1}\left\|T_{\alpha} f\right\|_{L^{p}(d \lambda)}\left\|K^{-1} \widetilde{g}_{\alpha}\right\|_{L^{q}(d \lambda)} .
$$

By Corollary 20, we have

$$
\left|\left\langle f, K^{-1} \tilde{g}\right\rangle_{\lambda}\right| \leq \mathrm{C} \sum_{|\alpha|=n+1}\left\|T_{\alpha} f\right\|_{L^{p}(d \lambda)}\left\|K^{-1} \tilde{g}\right\|_{L^{q}(d \lambda)},
$$

completing the proof of Theorem 18 .

Corollary 21. Suppose $p \geq 1$ and $\phi$ is holomorphic on $B_{n}$, then

(1) $h_{\bar{\phi}}$ is bounded $\Leftrightarrow T_{\alpha} \phi \in B \mathbb{C}$ for all $|\alpha|=n+1$;

(2) $h_{\bar{\phi}}$ is compact $\Leftrightarrow T_{\alpha} \phi \in \mathbb{C}_{0}$ for all $|\alpha|=n+1$;

(3) $h_{\bar{\phi}}$ is in $S_{p} \Leftrightarrow T_{\alpha} \phi \in L^{p}(d \lambda)$ for all $|\alpha|=n+1$, 
where

$$
T_{\alpha} \phi(z)=\left(1-|z|^{2}\right)^{n+1} \frac{\partial^{n+1} \phi}{\partial z^{\alpha}}(z)
$$

\section{THE POLY-DISC $\mathbb{D}^{n}$}

In this section we compute $V f$ for holomorphic functions $f$ on the polydisc $\mathbb{D}^{n}$ in $\mathbb{C}^{n}$. For simplicity of notation we restrict our computation to the bidisc $\mathbb{D}^{2}$ in $\mathbb{C}^{2} . \mathbb{D}$ will denote the open unit disc in $\mathbb{C}$.

Recall that for $f$ holomorphic in $\mathbb{D}^{2}$ we have

$$
\begin{gathered}
T_{1} f\left(z_{1}\right)=\left(1-\left|z_{1}\right|^{2}\right)^{2} \frac{\partial^{2} f}{\partial^{-2} z_{1}}(z, 0), \quad T_{2} f\left(z_{2}\right)=\left(1-\left|z_{2}\right|^{2}\right)^{2} \frac{\partial^{2} f}{\partial z_{2}^{2}}\left(0, z_{2}\right), \\
T_{3} f\left(z_{1}\right)=\left(1-\left|z_{1}\right|^{2}\right)^{2} \frac{\partial^{3} f}{\partial z_{1}^{2} \partial z_{2}}\left(z_{1}, 0\right), \quad T_{4} f\left(z_{2}\right)=\left(1-\left|z_{2}\right|^{2}\right)^{2} \frac{\partial^{3} f}{\partial z_{1} \partial z_{2}^{2}}\left(0, z_{2}\right), \\
T f\left(z_{1}, z_{2}\right)=\left(1-\left|z_{1}\right|^{2}\right)^{2}\left(1-\left|z_{2}\right|^{2}\right)^{2} \frac{\partial^{4} f}{\partial z_{1}^{2} \partial z_{2}^{2}}\left(z_{1}, z_{2}\right) .
\end{gathered}
$$

We will show that the above five functions describe the behaviour of $V f$. As a motivation, we first compare $V f$ with $T f$. Writing $f=P f$ and differentiating under integral sign, we easily get

$$
T f(z)=36\left(1-\left|z_{1}\right|^{2}\right)^{2}\left(1-\left|z_{2}\right|^{2}\right)^{2} \int_{\mathbb{D}} \frac{\bar{w}_{1}^{2} \bar{w}_{2}^{2} f\left(w_{1}, w_{2}\right)}{\left(1-z_{1} \bar{w}_{1}\right)^{4}\left(1-z_{2} \bar{w}_{2}\right)^{4}} d V(w) \text {. }
$$

By definition, we also have

$$
V f(z)=\lambda_{\mathbb{D}^{2}}\left(1-\left|z_{1}\right|^{2}\right)^{2}\left(1-\left|z_{2}\right|^{2}\right)^{2} \int_{\mathbb{D}^{2}} \frac{f\left(w_{1}, w_{2}\right)}{\left(1-z_{1} \bar{w}_{1}\right)^{4}\left(1-z_{2} \bar{w}_{2}\right)^{4}} d V(w),
$$

where $\lambda_{\mathbb{D}^{2}}=3^{2}=9$. Thus the only difference between $T f$ and $V f$ in the integral representation is the factor $\bar{w}_{1}^{2} \bar{w}_{2}^{2}$ in the integrand. Therefore, $T f$ and $V f$ are really closely related. On the other hand, $T f$ does not contain all the properties of $V f$. For example, if $f\left(z_{1}, z_{2}\right)$ only depends on one variable, say, $f\left(z_{1}, z_{2}\right)=g\left(z_{1}\right)$, then $T f=0$, but

$$
V f\left(z_{1}, z_{2}\right)=9\left(1-\left|z_{1}\right|^{2}\right)^{2}\left(1-\left|z_{2}\right|^{2}\right) \int_{\mathbb{D}} \frac{g\left(w_{1}\right)}{\left(1-z_{1} \bar{w}_{1}\right)^{4}} d V\left(w_{1}\right) .
$$

If $g$ is not in the Bloch space of $\mathbb{D}$, then $V f\left(z_{1}, z_{2}\right)$ is not even bounded, although $T f \equiv 0$. This explains why we need the functions $T_{i} f \quad(1 \leq i \leq 4)$.

We point out that if we are only concerned with boundedness, compactness, or membership in $S_{p}$ with $p>1$, then it is sufficient to consider the following three lower order derivatives [10, 12]:

$$
\begin{gathered}
\left(1-\left|z_{1}\right|^{2}\right) \frac{\partial f}{\partial z_{1}}\left(z_{1}, 0\right), \quad\left(1-\left|z_{1}\right|^{2}\right) \frac{\partial f}{\partial z_{2}}\left(0, z_{2}\right), \\
\left(1-\left|z_{1}\right|^{2}\right)\left(1-\left|z_{2}\right|^{2}\right) \frac{\partial^{2} f}{\partial z_{1} \partial z_{2}}\left(z_{1}, z_{2}\right) .
\end{gathered}
$$


In order to cover the endpoint $p=1$, we need all the five functions: $T_{i} f \quad(1 \leq$ $i \leq 4)$ and $T f$.

Theorem 22. Suppose $p \geq 1$ and $f$ is holomorphic in $\mathbb{D}^{2}$, we have

(1) $V f \in B \mathbb{C} \Leftrightarrow T_{i} f \in B \mathbb{C}(\mathbb{D})(1 \leq i \leq 4), T f \in B \mathbb{C}\left(\mathbb{D}^{2}\right)$;

(2) $V f \in \mathbb{C}_{0} \Leftrightarrow T_{i} f \in \mathbb{C}_{0}(\mathbb{D})(1 \leq i \leq 4), T f \in \mathbb{C}_{0}\left(\mathbb{D}^{2}\right)$;

(3) $V f \in L^{p}(d \lambda) \Leftrightarrow T_{i} f \in L^{p}(\mathbb{D}, d \lambda)(1 \leq i \leq 4), T f \in L^{p}\left(\mathbb{D}^{2}, d \lambda\right)$.

Combining Theorems 8, 12, 15 and 22, we get

Corollary 23. Suppose $p \geq 1$ and $\phi$ is holomorphic in $\mathbb{D}^{2}$, then we have

(1) $h_{\bar{\phi}}$ is bounded $\Leftrightarrow T_{i} \phi \in B \mathbb{C}(\mathbb{D})(1 \leq i \leq 4), T \phi \in B \mathbb{C}\left(\mathbb{D}^{2}\right)$;

(2) $h_{\bar{\phi}}$ is compact $\Leftrightarrow T_{i} \phi \in \mathbb{C}_{0}(\mathbb{D})(1 \leq i \leq 4), T \phi \in \mathbb{C}_{0}\left(\mathbb{D}^{2}\right)$;

(3) $h_{\bar{\phi}}$ is in $S_{p} \Leftrightarrow T_{i} \phi \in L^{p}(\mathbb{D}, d \lambda)(1 \leq i \leq 4), T \phi \in L^{p}\left(\mathbb{D}^{2}, d \lambda\right)$.

The proof of Theorem 22 is similar to that of Theorem 18 .

Once again the three implications " $\Rightarrow$ " are easier to prove. Given $f$ holomorphic in $\mathbb{D}^{2}$ with $V f \in B \mathbb{C}$. Write $f=P V f$ and differentiate under integral sign, then

$$
T f(z)=36\left(1-\left|z_{1}\right|^{2}\right)^{2}\left(1-\left|z_{2}\right|^{2}\right)^{2} \int_{\mathbb{D}^{2}} \frac{\bar{w}_{1}^{2} \bar{w}_{2}^{2} V f\left(w_{1}, w_{2}\right)}{\left(1-z_{1} \bar{w}_{1}\right)^{4}\left(1-z_{2} \bar{w}_{2}\right)^{4}} d V(w) .
$$

Let $M$ be the operator defined by

$$
M f(z)=4 \bar{z}_{1}^{2} \bar{z}_{2}^{2} f(z)
$$

then we have

$$
T f=V M V f \text {. }
$$

$M$ maps $B \mathbb{C}$ into $B \mathbb{C}, \mathbb{C}_{0}$ into $\mathbb{C}_{0}$, and $L^{p}(d \lambda)$ into $L^{p}(d \lambda)$, and so does $V$, thus $V f \in B \mathbb{C}$ implies $T f \in B \mathbb{C}, V f \in \mathbb{C}_{0}$ implies $T f \in \mathbb{C}_{0}$, and $V f \in L^{p}(d \lambda)$ implies $T f \in L^{p}(d \lambda)$.

To get the same conclusions about $T_{1} f$ and $T_{2} f$, notice that $f=P V f$ gives

$$
T_{1} f\left(z_{1}\right)=6 \int_{\mathbb{D}^{2}} \frac{\left(1-\left|z_{1}\right|^{2}\right)^{2} \bar{w}_{1}^{2} V f\left(w_{1}, w_{2}\right)}{\left(1-z_{1} \bar{w}_{1}\right)^{4}} d V(w) .
$$

Let

$$
\phi_{1}\left(w_{1}\right)=\bar{w}_{1}^{2} \int_{\mathbb{D}} V f\left(w_{1}, w_{2}\right) d V\left(w_{2}\right)
$$

then

$$
T_{1} f\left(z_{1}\right)=6\left(1-\left|z_{1}\right|^{2}\right)^{2} \int_{\mathbb{D}} \frac{\phi\left(w_{1}\right) d V\left(w_{1}\right)}{\left(1-z_{1} \bar{w}_{1}\right)^{4}}
$$

with

$$
\left|\phi_{1}\left(z_{1}\right)\right|^{p} \leq \int_{\mathbb{D}}\left|V f\left(z_{1}, z_{2}\right)\right|^{p} d V\left(z_{2}\right) \leq \int_{\mathbb{D}} \frac{\left|V f\left(z_{1}, z_{2}\right)\right|^{p}}{\left(1-\left|z_{2}\right|^{2}\right)^{2}} d V\left(z_{2}\right)
$$


and

$$
\int_{\mathbb{D}} \frac{\left|\phi_{1}\left(z_{1}\right)\right|^{p}}{\left(1-\left|z_{1}\right|^{2}\right)^{2}} d V\left(z_{1}\right) \leq \int_{\mathbb{D}} \int_{\mathbb{D}} \frac{\left|V f\left(z_{1}, z_{2}\right)\right|^{p} d V(z)}{\left(1-\left|z_{1}\right|^{2}\right)^{2}\left(1-\left|z_{2}\right|^{2}\right)^{2}}
$$

or

$$
\left\|\phi_{1}\right\|_{L^{p}(\mathbb{D}, d \lambda)} \leq\|V f\|_{L^{p}\left(\mathbb{D}^{2}, d \lambda\right)} .
$$

Thus the result on $\mathbb{D}=B_{1}$ implies that $T_{1} f \in L^{p}(\mathbb{D}, d \lambda)$ if $V f \in L^{p}\left(\mathbb{D}^{2}, d \lambda\right)$. Similarly, $T_{1} f \in \mathbb{C}_{0}(\mathbb{D})$ if $V f \in \mathbb{C}_{0}\left(\mathbb{D}^{2}\right), T_{1} f \in B \mathbb{C}(D)$ if $V f \in B \mathbb{C}\left(\mathbb{D}^{2}\right)$. The case $T_{2} f$ can be treated similarly.

To get the results for $T_{3} f$ and $T_{4} f$, we write $f=P V f$ and differentiate under integral sign, then we get

$$
T_{3} f\left(z_{1}\right)=12\left(1-\left|z_{1}\right|^{2}\right)^{2} \int_{\mathbb{D}^{2}} \frac{\bar{w}_{1}^{2} \bar{w}_{2} V f\left(w_{1}, w_{2}\right)}{\left(1-z_{1} \bar{w}_{1}\right)^{4}} d V(w) \text {. }
$$

Let

$$
\psi_{1}\left(w_{1}\right)=\bar{w}_{1}^{2} \int_{\mathbb{D}} \bar{w}_{2} V f\left(w_{1}, w_{2}\right) d V\left(w_{2}\right)
$$

then

$$
T_{3} f\left(z_{1}\right)=12\left(1-\left|z_{1}\right|^{2}\right)^{2} \int_{\mathbb{D}} \frac{\psi_{1}\left(w_{1}\right)}{\left(1-z_{1} \bar{w}_{1}\right)^{4}} d V\left(w_{1}\right)
$$

and $\psi_{1}$ satisfies the same estimate we did for $\phi_{1}$, thus the result on $\mathbb{D}=B_{1}$ gives the implication that $V f \in B \mathbb{C}\left(\mathbb{D}^{2}\right) \Leftrightarrow T_{3} f \in B \mathbb{C}(\mathbb{D}), V f \in \mathbb{C}_{0}\left(\mathbb{D}^{2}\right) \Leftrightarrow$ $T_{3} f \in \mathbb{C}_{0}(\mathbb{D})$, and $V f \in L^{p}\left(\mathbb{D}^{2}, \lambda\right) \Leftrightarrow T_{3} f \in L^{p}(\mathbb{D}, d \lambda)$. The same proof works for $T_{4} f$. We have completed the proof for the three implications " $\Rightarrow$ " in Theorem 22.

We will prove the converse only for (3). The other two statements can be proved similarly and details can be found in $[10,12]$.

Given $f$ holomorphic in $\mathbb{D}^{2}$ with $f(0)=0$, we can always write

$$
f\left(z_{1}, z_{2}\right)=h_{1}\left(z_{1}\right)+h_{2}\left(z_{2}\right)+z_{1} z_{2}\left(h_{3}\left(z_{1}\right)+h_{4}\left(z_{2}\right)\right)+z_{1}^{2} z_{2}^{2} h\left(z_{1}, z_{2}\right) .
$$

Moreover, the decomposition is unique if $h_{4}(0)=0$. Since $f(0)=0$, we have $h_{1}\left(z_{1}\right)=f\left(z_{1}, 0\right), h_{2}\left(z_{2}\right)=f\left(0, z_{2}\right)$. Therefore,

$$
\begin{aligned}
V h_{1}\left(z_{1}, z_{2}\right) & =9\left(1-\left|z_{1}\right|^{2}\right)^{2}\left(1-\left|z_{2}\right|^{2}\right)^{2} \int_{\mathbb{D}^{2}} \frac{h_{1}\left(w_{1}\right) d V(w)}{\left(1-z_{1} \bar{w}_{1}\right)^{4}\left(1-z_{2} \bar{w}_{2}\right)^{4}} \\
& =9\left(1-\left|z_{2}\right|^{2}\right)^{2}\left(1-\left|z_{1}\right|^{2}\right)^{2} \int_{\mathbb{D}} \frac{f_{1}\left(w_{1}, 0\right) d V\left(w_{1}\right)}{\left(1-z_{1} \bar{w}_{1}\right)^{4}} .
\end{aligned}
$$

The assumption that $T_{1} f \in L^{p}(\mathbb{D}, d \lambda)$ together with the results for $\mathbb{D}=B_{1}$ now gives $V h_{1} \in L^{p}\left(\mathbb{D}^{2}, d \lambda\right)$. Similarly, $T_{2} f \in L^{p}(\mathbb{D}, d \lambda) \Leftrightarrow V h_{2} \in L^{p}\left(\mathbb{D}^{2}, d \lambda\right)$.

It is easy to see that

$$
\frac{\partial f}{\partial z_{2}}\left(z_{1}, 0\right)=h_{2}^{\prime}(0)+z_{1} h_{3}\left(z_{1}\right)
$$


or

$$
z_{2} \frac{\partial f}{\partial z_{2}}\left(z_{1}, 0\right)=h_{2}^{\prime}(0) z_{2}+z_{1} z_{2} h_{3}\left(z_{1}\right)
$$

It follows that

$$
V\left(z_{1} z_{2} h_{3}\left(z_{1}\right)\right)\left(z_{1}, z_{2}\right)=V\left(z_{2} \frac{\partial f}{\partial z_{2}}\left(z_{1}, 0\right)\right)\left(z_{1}, z_{2}\right)-h_{2}^{\prime}(0) V\left(z_{2}\right)\left(z_{1}, z_{2}\right) \text {. }
$$

Clearly, $V\left(z_{2}\right)\left(z_{1}, z_{2}\right) \in L^{p}(d \lambda)$. Also we have

$$
\begin{aligned}
& V\left(z_{2} \frac{\partial f}{\partial z_{2}}\left(z_{1}, 0\right)\right)\left(z_{1}, z_{2}\right) \\
& =9\left(1-\left|z_{1}\right|^{2}\right)^{2}\left(1-\left|z_{2}\right|^{2}\right)^{2} \int_{\mathbb{D}^{2}} \frac{\frac{\partial f}{\partial w_{2}}\left(w_{1}, 0\right) w_{2} d V(w)}{\left(1-z_{1} \bar{w}_{1}\right)^{4}\left(1-z_{2} \bar{w}_{2}\right)^{4}} \\
& =18 z_{2}\left(1-\left|z_{2}\right|^{2}\right)^{2}\left(1-\left|z_{1}\right|^{2}\right)^{2} \int_{\mathbb{D}^{2}} \frac{\frac{\partial f}{\partial w_{2}}\left(w_{1}, 0\right)}{\left(1-z_{1} \bar{w}_{1}\right)^{4}} d V\left(w_{1}\right) .
\end{aligned}
$$

So $T_{3} f \in L^{p}(\mathbb{D}, d \lambda)$ together with the results on $\mathbb{D}=B_{1}$ gives

$$
V\left(z_{2} \frac{\partial f}{\partial z_{2}}\left(z_{1}, 0\right)\right)\left(z_{1}, z_{2}\right) \in L^{p}\left(\mathbb{D}^{2}, d \lambda\right) \text {. }
$$

Therefore,

$$
V\left(z_{1} z_{2} h_{3}\left(z_{1}\right)\right) \in L^{p}\left(\mathbb{D}^{2} d \lambda\right) .
$$

Similarly,

$$
V\left(z_{1} z_{2} h_{4}\left(z_{2}\right)\right) \in L^{p}\left(\mathbb{D}^{2}, d \lambda\right) .
$$

It remains to show that

$$
V\left(z_{1}^{2} z_{2}^{2} h\left(z_{1}, z_{2}\right)\right) \in L^{p}\left(\mathbb{D}^{2}, d \lambda\right) .
$$

In doing this we may as well assume that

$$
f\left(z_{1}, z_{2}\right)=z_{1}^{2} z_{2}^{2} h\left(z_{1}, z_{2}\right) \text {. }
$$

Given $g \in L^{q}(d \lambda)$ with $1 / p+1 / q=1$, then

$$
\langle V f, g\rangle_{\lambda}=\langle f, V g\rangle_{\lambda} .
$$

Write $V g(z)=K(z, z)^{-1} \tilde{g}(z)$ with

$$
\tilde{g}(z)=\tilde{g}(0)+\tilde{h}_{1}\left(z_{1}\right)+\widetilde{h}_{2}\left(z_{2}\right)+z_{1} z_{2}\left(\widetilde{h}_{3}\left(z_{1}\right)+\widetilde{h}_{4}\left(z_{2}\right)\right)+z_{1}^{2} z_{2}^{2} \widetilde{h}\left(z_{1}, z_{2}\right) .
$$

The unitary invariance of $K^{-1}$ and $d \lambda$ now implies that

$$
\begin{aligned}
\langle f, V g\rangle_{\lambda} & =\left\langle f(z), K(z, z)^{-1} z_{1}^{2} z_{2}^{2} \widetilde{h}\left(z_{1}, z_{2}\right)\right\rangle_{\lambda} \\
& =\left\langle\bar{z}_{1}^{2} \bar{z}_{1}^{2} f(z), K(z, z)^{-1} \tilde{h}(z)\right\rangle_{\lambda} .
\end{aligned}
$$

Because $K^{-1} \tilde{h}$ is in the image of $V$ and $V$ is a projection in the pairing $\langle,\rangle_{\lambda}$ 
thus

$$
\begin{aligned}
\langle f, V g\rangle_{\lambda} & =\left\langle V\left(\bar{z}_{1}^{2} \bar{z}_{1}^{2} f(z)\right), K^{-1}(z, z) \tilde{h}(z)\right\rangle_{\lambda} \\
& =\frac{1}{4}\left\langle T f, K^{-1} \tilde{h}\right\rangle_{\lambda} .
\end{aligned}
$$

It follows that

$$
\left|\langle f, V g\rangle_{\lambda}\right| \leq \frac{1}{4}\|T f\|_{L^{p}(d \lambda)}\left\|K^{-1} \widetilde{h}\right\|_{L^{q}(d \lambda)} .
$$

The desired result now follows from the following.

Lemma 24. Suppose $g$ is holomorphic in $\mathbb{D}^{2}$ with $g(0)=0$ and

$$
g\left(z_{1} z_{2}\right)=h_{1}\left(z_{1}\right)+h_{2}\left(z_{2}\right)+z_{1} z_{2}\left(h_{3}\left(z_{1}\right)+h_{4}\left(z_{2}\right)\right)+z_{1}^{2} z_{2}^{2} h\left(z_{1} z_{2}\right) \text {, }
$$

then

$$
\left\|K^{-1} h\right\|_{L^{p}(d \lambda)} \leq C\left\|K^{-1} g\right\|_{L^{p}(d \lambda)}
$$

with $C$ independent of $g$.

Proof. Since $h_{1}\left(z_{1}\right)=g\left(z_{1}, 0\right)$, thus

$$
\left\|K^{-1} h_{1}\right\|_{L^{p}(d \lambda)} \leq\left\|K^{-1} g\right\|_{L^{p}(d \lambda)} \text {. }
$$

Similarly, $\left\|K^{-1} h_{2}\right\|_{L^{p}(d \lambda)} \leq\left\|K^{-1} g\right\|_{L^{p}(d \lambda)}$. Next write

$$
\tilde{g}\left(z_{1}, z_{2}\right)=\frac{g\left(z_{1}, z_{2}\right)-h_{1}\left(z_{1}\right)-h_{2}\left(z_{2}\right)}{z_{1} z_{2}}=h_{3}\left(z_{1}\right)+h_{4}\left(z_{2}\right)+z_{1} z_{2} h\left(z_{1} z_{2}\right) \text {. }
$$

Using Lemma 15 in [10] (or a similar version of it), we can get

$$
\left\|K^{-1} \tilde{g}\right\|_{L^{p}(d \lambda)} \leq C_{1}\left\|K^{-1} g\right\|_{L^{p}(d \lambda)} \text {. }
$$

Repeating the above process one more time, we get

$$
\left\|K^{-1} h\right\|_{L^{p}(d \lambda)} \leq C_{2}\left\|K^{-1} g\right\|_{L^{p}(d \lambda)} .
$$

\section{REFERENCES}

1. J. Arazy, S. Fisher, and J. Peetre, Hankel operators on weighted Bergman spaces, Amer. J. Math. 110 (1988), 989-1054.

2. C. A. Berger, L. A. Coburn, and K. H. Zhu, Function theory on Cartan domains and the Berezin-Toeplitz symbol calculus, Amer. J. Math. 110 (1988), 921-953.

3. D. Békollé, C. A. Berger, L. A. Coburn, and K. H. Zhu, BMO in the Bergman metric on bounded symmetric domains, J. Funct. Anal. (to appear).

4. R. Coifman and R. Rochberg, Representation theorems for holomorphic and harmonic functions in $L^{p}$, Astérisque 77 (1980), 11-66.

5. I. Gohberg and M. G. Krein, Introduction to the theory of nonselfadjoint operators, Amer. Math. Soc. Transl. 18 (1969).

6. S. Janson, J. Peetre, and R. Rochberg, Hankel forms and the Fock space, Revista Mat. Ibero-Aerm. 3 (1987), 61-138.

7. D. Luecking, Trace ideal criteria for Toeplitz operators, J. Funct. Anal. 73 (1987), 345-368.

8. W. Rudin, Function theory in the unit ball of $\mathbb{C}^{n}$, Springer-Verlag, New York and Berlin, 1980.

9. M. Stoll, Mean value theorems for harmonic and holomorphic functions on bounded symmetric domains, J. Reine Angew. Math. 290 (1977), 191-198. 
10. K. H. Zhu, Duality and Hankel operators on the Bergman space of bounded symmetric domains, J. Funct. Anal. 81 (1988), 260-278.

11. _ Positive Toeplitz operators on weighted Bergman spaces of bounded symmetric domains, J. Operator Theory 20 (1988), 329-357.

12. _ The Bergman spaces, the Bloch space, and Gleason's problem, Trans. Amer. Math. Soc. 309 (1988), 253-268.

Department of Mathematics, State University of New York, Albany, New York 12222 